\title{
Perforación del yeyuno por enfermedad diverticular: una condición a considerar en ancianos
}

\section{Perforation of the jejunum due to diverticular disease: A condition to consider in the elderly}

Carlos Mario Balcázar V., ${ }^{\text {* }}$ Beatriz Carolina Ayola R., ${ }^{2}$ José Mauricio Ocampo Ch., ${ }^{3}$ Mónica Bejarano, MSc, MACC. ${ }^{4}$

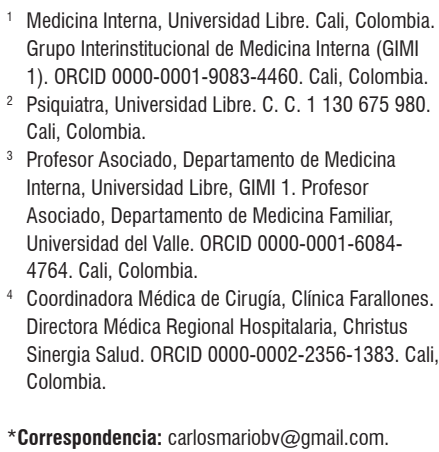

Medicina Interna, Universidad Libre. Cali, Colombia. Grupo Interinstitucional de Medicina Interna (GIMI 1). ORCID 0000-0001-9083-4460. Cali, Colombia.

2 Psiquiatra, Universidad Libre. C. C. 1130675980. Cali, Colombia.

3 Profesor Asociado, Departamento de Medicina Interna, Universidad Libre, GIMI 1. Profesor Asociado, Departamento de Medicina Familiar, Universidad del Valle. ORCID 0000-0001-60844764. Cali, Colombia

${ }^{4}$ Coordinadora Médica de Cirugía, Clínica Farallones. Directora Médica Regional Hospitalaria, Christus Sinergia Salud. ORCID 0000-0002-2356-1383. Cali, Colombia.

*Correspondencia: carlosmariobv@gmail.com.

\begin{abstract}
Resumen
La enfermedad diverticular constituye la principal afección intestinal después de los 40 años; tiene gran relevancia por sus amplias manifestaciones, que llevan a consultas frecuentes en todos los servicios de urgencias y corresponden al hallazgo más usual en los procedimientos endoscópicos electivos. Por su parte, la diverticulosis del intestino delgado tiene una prevalencia que oscila entre $2 \%$ y $5 \%$. Las presentaciones clínicas como sangrado, obstrucción, dolor abdominal, perforación, formación de abscesos y fístulas suelen ser más floridas cuando afectan el colon. Se presenta el caso de un paciente anciano quien consultó a urgencias con abdomen agudo secundario a peritonitis generalizada por perforación intestinal debida a enfermedad diverticular del yeyuno.
\end{abstract}

\section{Palabras clave \\ Anciano, geriatría, intestino, enfermedades del yeyuno, divertículo, delirio.}

\begin{abstract}
Diverticular disease is the most common bowel disease after the age of 40 years. It is the most common finding in elective endoscopic procedures, and it has great relevance because of its broad manifestations which lead to frequent emergency service consultations. On the other hand, the prevalence of diverticulosis of the small intestine ranges from $2 \%$ to $5 \%$. Clinical presentations such as bleeding, obstructions, abdominal pain, perforations, formation of abscesses and fistulas are usually more florid when they affect the colon. We present the case of an elderly emergency room patient with acute abdomen secondary to generalized peritonitis due to intestinal perforation caused by diverticular disease of the jejunum.
\end{abstract}

Keywords

Elderly patients, geriatrics, intestine, diseases of the jejunum, diverticulum, delirium.

\section{INTRODUCCIÓN}

Los divertículos son formaciones saculares producidas por la protrusión de mucosa a través de la pared muscular del intestino, cuya prevalencia es similar en hombres y mujeres (1), pero aumenta con la edad, desde menos del $20 \%$ a los 40 años hasta más de $63 \%$ en mayores de 70 años (2). La enfermedad diverticular afecta principalmente el colon, sobre todo el sigmoides (2). La presencia de divertículos en el intestino delgado es menos frecuente; sin embargo, el $80 \%$ de la diverticulosis del intestino delgado ocurre en el yeyuno, $15 \%$ en el íleon y $5 \%$ en ambos (3), y en series de necropsias se ha reportado a nivel del yeyuno e íleon entre el $1 \%$ y $4,5 \%(4,5)$.

La etiología de los divertículos yeyunales no es clara, pero se han considerado alteraciones de la motilidad (6) y existen factores de riesgo nutricionales que incrementan la formación de divertículos en general, como la dieta baja 
en fibra y rica en azúcares refinados (7). El escaso aporte de fibra resulta en heces poco hidratadas, las cuales pueden alterar el tiempo de tránsito intestinal, factor que se traduce en el incremento en la presión intracolónica, lo que dificulta la evacuación del contenido intestinal y favorece la formación de divertículos (7). Además, se han descrito otros factores de riesgo que pueden aumentar su frecuencia de aparición, como el cambio en la microbiota, estreñimiento, sedentarismo, obesidad, tabaquismo y consumo de antinflamatorios no esteroideos (AINE) (2).

La enfermedad diverticulares asintomática hasta en el $75 \%$ de los casos. Los síntomas más frecuentes en general son: dolor abdominal (8), sangrado digestivo bajo e inflamación con formación de abscesos, perforaciones u obstrucción (5). En el intestino delgado el diagnóstico puede ser difícil porque no hay características patognomónicas ni síntomas específicos (9).

Por lo anterior, es de vital importancia conocer las formas de presentación de la enfermedad diverticular atípica en el paciente anciano, porque el diagnóstico oportuno va a tener impacto en la supervivencia del paciente.

\section{DESCRIPCIÓN DEL CASO}

Es un hombre de 67 años que consultó por cuadro de dos días de evolución de dolor abdominal tipo punzada, de intensidad moderada e inicio gradual, localizado en el hipogastrio, irradiado al mesogastrio y la fosa ilíaca derecha, acompañado de distensión abdominal, emesis posprandial, dos episodios de deposiciones diarreicas (sin moco, ni sangre) y fiebre no cuantificada; había presentado fluctuación del estado de conciencia, desorientación y aparición de alucinaciones visuales zoomorfas.

El único antecedente comórbido era hipertensión arterial crónica, controlado con un inhibidor de la enzima convertidora de angiotensina (IECA); no tenía antecedentes quirúrgicos, historia de compromiso cognitivo, ni enfermedad mental y con condición funcional previa al ingreso independiente para realizar sus actividades básicas e instrumentales de la vida diaria (escala de Barthel: 100/100).

En la exploración física y mental al ingreso se encontró al paciente letárgico, desorientado temporo-espacialmente, con fluctuación del estado de conciencia; no logró sostener una conversación fluida, tuvo pensamiento irrelevante, lenguaje incoherente, febril (temperatura: $38,8^{\circ} \mathrm{C}$ ), taquicárdico (frecuencia cardíaca [FC]: 120 latidos por minuto $[\mathrm{lpm}]$ ), polipneico (frecuencia respiratoria [FR]: 24 respiraciones por minuto $[\mathrm{rpm}]$ ) e hipotenso (tensión arterial [TA]: 90/50 mm Hg) con llenado capilar de 4 segundos. El abdomen estaba marcadamente distendido con ausencia de peristaltismo, dolor a la palpación superficial en flanco y fosa ilíaca derecha, había timpanismo central y defensa abdominal voluntaria.

La impresión diagnóstica clínica inicial fue de sepsis de origen abdominal secundaria a apendicitis aguda, acompañada de delirio hipoactivo, teniendo en cuenta la presencia de un quick Sequential Organ Failure Assessment score (qSOFA) de 3 puntos (10) y las manifestaciones psiquiátricas evidentes al momento del interrogatorio, que se correlacionaron con un Short Confusion Assessment Method (Short-CAM) de comienzo agudo y curso fluctuante del estado mental.

Se inició el manejo con líquidos intravenosos (y se logró normalidad hemodinámica) y cubrimiento antibiótico a base de ampicilina/sulbactam. Se solicitaron estudios paraclínicos que mostraron un hemograma con leucocitos: $15530 / \mu \mathrm{L}$, hemoglobina: $10,4 \mathrm{~g} / \mathrm{dL}$ plaquetas: $130000 / \mu \mathrm{L}$, además de proteína C-reactiva (PCR): $114 \mathrm{mg} / \mathrm{dL}$, gases arteriales con pH: 7,31, presión parcial de dióxido de carbono $\left(\mathrm{pCO}_{2}\right): 22,7 \mathrm{~mm} \mathrm{Hg}$, presión parcial de oxígeno $\left(\mathrm{PO}_{2}\right): 70,3 \mathrm{~mm} \mathrm{Hg}$, bicarbonato $\left(\mathrm{HCO}_{3}\right): 11,2 \mathrm{mmol} / \mathrm{L}$ y base exceso (BE): $-12,7 \mathrm{mmol} / \mathrm{L}$. Se realizaron radiografías de tórax de pie (Figura 1) y de abdomen simple (Figura 2).

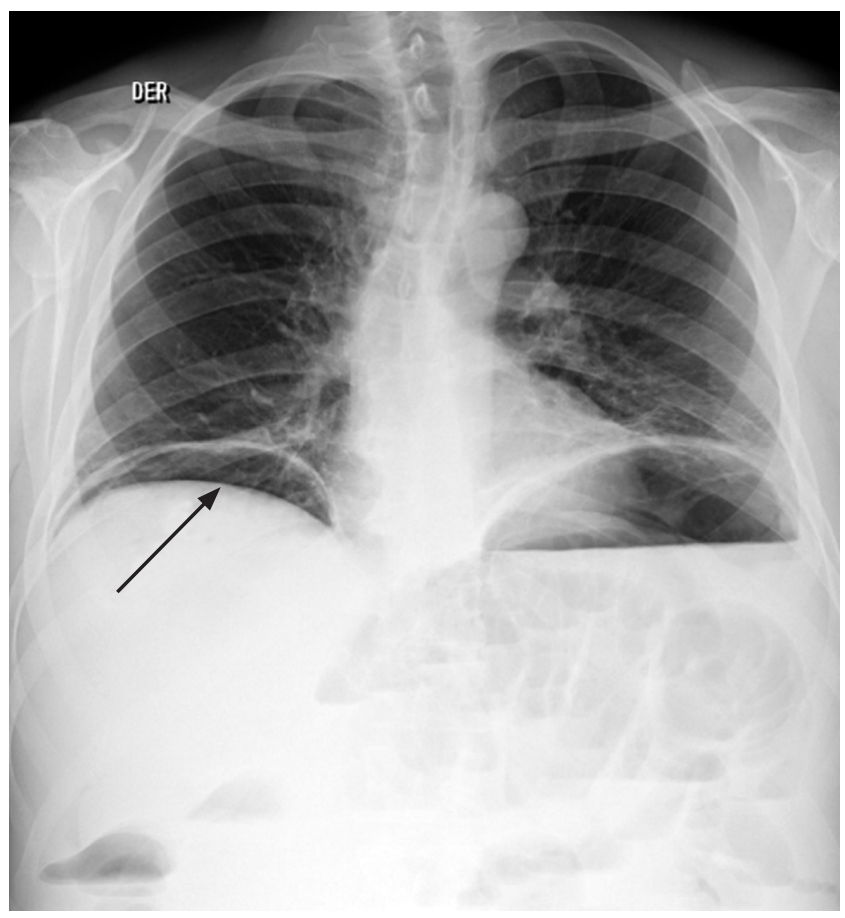

Figura 1. Radiografía de tórax posteroanterior de pie. Se aprecia la imagen del neumoperitoneo bilateral (flecha) con colección subpulmonar basal bilateral con nivel hidroaéreo en la base izquierda acompañado de la elevación de ambos hemidiafragmas y opacidades intersticiales reticulares biliares bilaterales, sin derrames pleurales.

Con estos hallazgos se llevó a cirugía de emergencia para la realización de laparotomía exploratoria y se encontró una 
peritonitis de cuatro cuadrantes y un segmento de $10 \mathrm{~cm}$ de intestino delgado (yeyuno) de aspecto congestivo, edematoso y con áreas de necrosis, el cual se resecó. Se realizó anastomosis terminoterminal y lavado con solución salina tibia. El estudio del espécimen quirúrgico identificó la presencia de formaciones diverticulares con reacción inflamatoria (Figuras 3 y 4 ).

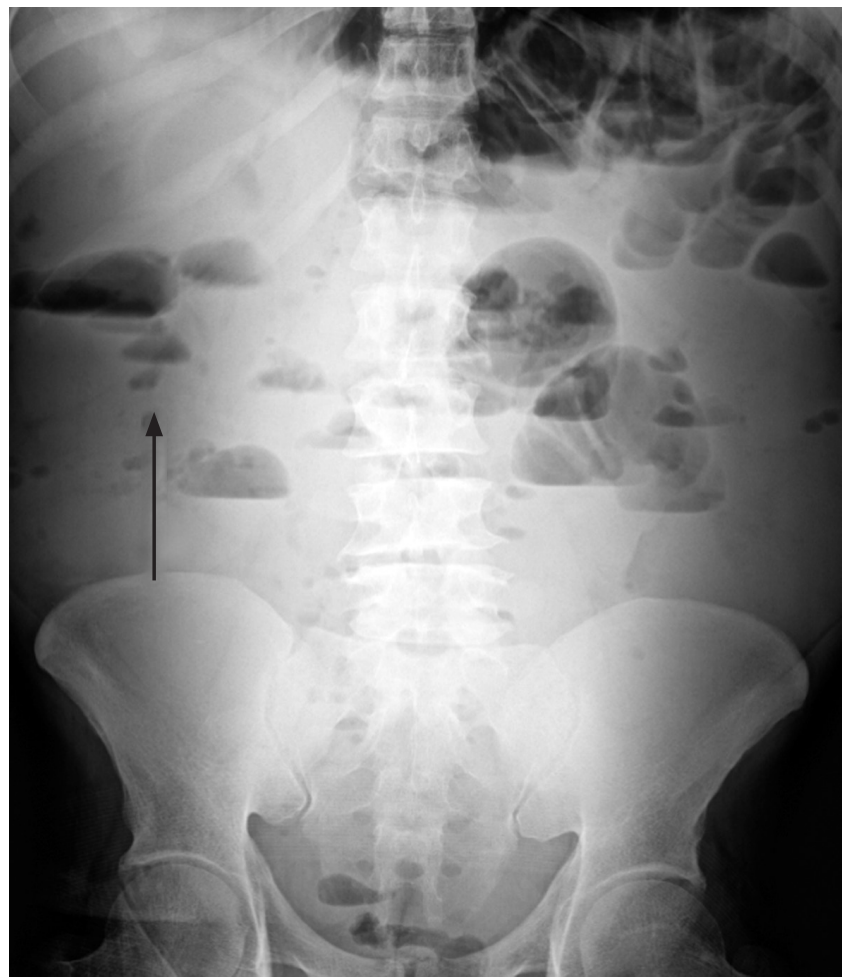

Figura 2. Radiografía simple de abdomen. Se aprecian múltiples niveles hidroaéreos (flecha) que comprometen toda la cavidad abdominal, sobre todo en el mesogastrio y epigastrio, con presencia de aire en la ampolla rectal (obstrucción parcial o fenómeno de íleo).

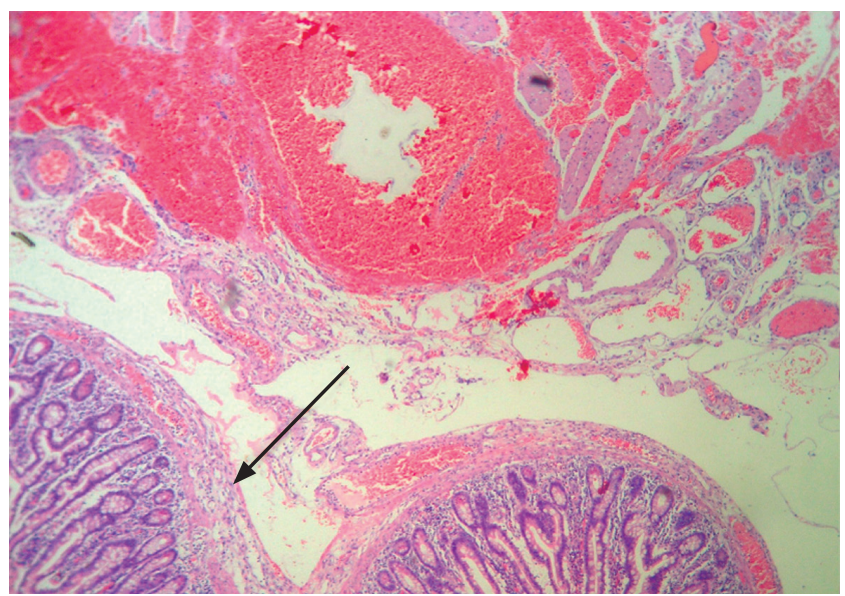

Figura 3. Segmento del yeyuno en el que se observa más evidentemente el edema e inflamación a través de toda la pared (flecha) en presencia de formaciones diverticulares (hematoxilina-eosina. Aumento: $50 \mathrm{X}$ ).

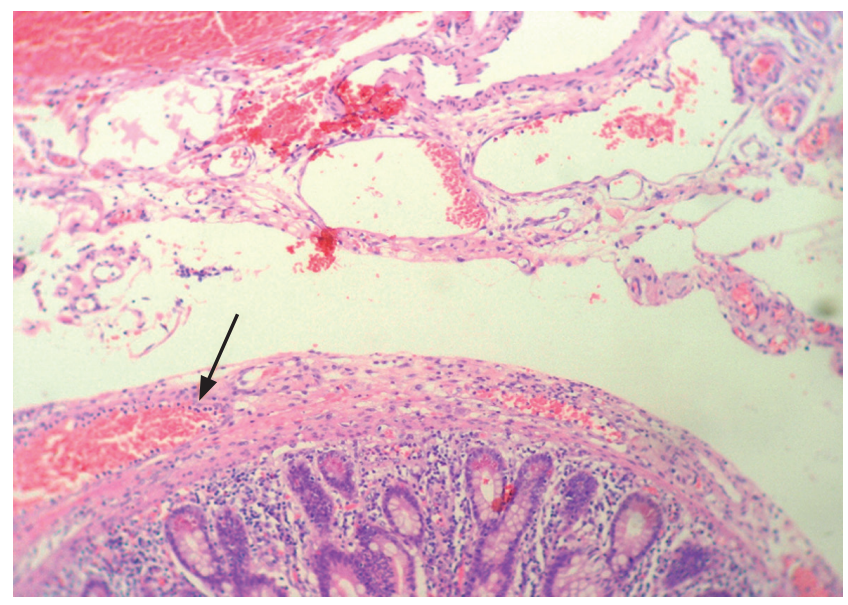

Figura 4. Segmento del intestino delgado en el que se observa un edema de la pared con un infiltrado inflamatorio que va desde la mucosa hasta la serosa; además, se observa un sangrado que se ve está más allá de la serosa (flecha), pero no adherido a ella, que probablemente esté en relación con el sangrado en el peritoneo (hematoxilina-eosina. Aumento: $100 \mathrm{X}$ ).

El abdomen inicialmente quedó en manejo con el sistema de presión negativa y se realizaron lavados quirúrgicos cada 48 horas hasta el cierre de la pared abdominal el día 10. El paciente se manejó en la unidad de cuidados intensivos (UCI) durante 6 días, luego siguió su evolución favorable en la sala general, con resolución de la sepsis abdominal, mejoría de su condición clínica, mental y recuperación de su funcionalidad para egreso el día 14.

\section{DISCUSIÓN}

El dolor abdominal agudo es causa de consulta frecuente en el servicio de urgencias, sobre todo en la población anciana, de los cuales un gran porcentaje requiere manejo intrahospitalario o quirúrgico (11); esto incrementa el costo del sistema de salud, más aún cuando no se logra hacer un diagnóstico rápido y una intervención oportuna.

Se presenta el caso de un adulto mayor, quien consultó por dolor abdominal agudo de características descritas, asociado con manifestaciones psiquiátricas compatibles con delirio. Clínicamente se encontraron datos de síndrome de respuesta inflamatoria sistémica (SIRS) con un qSOFA positivo $>2$ (10). Se tomaron estudios paraclínicos que reportaron reactantes inflamatorios altos y hemograma con desviación a la izquierda en el paciente con acidosis metabólica, lo que apoyó el diagnóstico de sepsis (10) y ameritó una intervención guiada por metas. Durante la observación se evidenció por imágenes la ruptura de una víscera hueca y signos de obstrucción intestinal parcial (12). Por la localización anatómica del dolor se sospechó patología apendicular; sin embargo, no se descartó la posi- 
bilidad de perforación espontánea del intestino delgado. Las causas de perforación espontánea del intestino delgado aparecen en la Tabla 1.

Tabla 1. Causas de perforación espontánea del intestino delgado (13)

\begin{tabular}{ll}
\hline \multicolumn{1}{c}{ Origen } & \multicolumn{1}{c}{ Enfermedad } \\
\hline Inflamatorio/ & Enfermedad diverticular (diverticulitis) (11) \\
obstructivo & Enfermedad de Crohn \\
Autoinmune & Enfermedad celíaca \\
& Enfermedad de injerto contra el huésped \\
Infeccioso & Viral: citomegalovirus \\
& Bacterias: Salmonella y Mycobacterium \\
& tuberculosis \\
& Parásitos: Ascaris lumbricoides \\
Inducido por & AlNE \\
agentes biológicos o & Quimioterapéuticos \\
medicamentos & Inmunobiológicos \\
Congénito & Divertículo de Meckel \\
& Duplicación de yeyuno o íleon \\
Metabólico & Homocisteinuria \\
Vascular & Poliangitis microscópica granulomatosa \\
& Arteritis de células gigantes \\
& Injuria vascular inducia por radiación \\
Neoplásico & Primaria: adenocarcinoma, otros \\
& Secundaria: melanoma, mesotelioma, otros \\
\hline
\end{tabular}

Adaptado de: Freeman HJ. World J Gastroenterol. 2014;20(29):9990-7.

Sin embargo, existe otra forma de enfocar el dolor abdominal agudo en el paciente anciano y se basa en la probable causa etiológica que se puede resumir en causas obstructivas, inflamatorias, vasculares o criptogénicas (14), que por la edad y la presentación del caso están a favor de una condición inflamatoria frente a vascular (15). Finalmente, en este caso la patología logró identificar la presencia de enfermedad diverticular grave de yeyuno con signos de perforación (Figuras 3 y 4 ).

Actualmente se sabe que la enfermedad diverticular del intestino delgado no tiene una etiología bien establecida y se han planteado múltiples hipótesis; fue descrita por primera vez en el 1794 por Sommering y casi 15 años más tarde confirmada por Sir Astley Cooper (16). La prevalencia ocurre entre los 47 y los 86 años, con promedio a los 72 (17) y la forma de presentación no es específica: va desde dolor abdominal difuso hasta complicaciones letales de todo tipo, más aún en el anciano, en quien puede debutar de forma atípica, como el delirio (18). Sin embargo, en la mayoría de los casos es asintomática (90\% cuando se afecta el duodeno y $40 \%$ el yeyuno). En este último grupo se torna sintomática en el $40 \%$ de casos y su principal manifestación es el síndrome de malabsorción intestinal (19).

Dentro de sus complicaciones más frecuentes se conoce el mayor riesgo de obstrucción intestinal y sangrado digestivo, que puede terminar en formación de abscesos y, en casos más infrecuentes, en fístulas y perforación espontánea como manifestación de abdomen agudo, como en este caso (20). Por esta razón, es importante ampliar los diagnósticos diferenciales y recordar que la presencia de divertículos del intestino delgado está siempre presente.

Para finalizar, lo interesante del caso es poder recordar que existen causas de abdomen agudo que no se tienen en cuenta al momento de valorar a los pacientes geriátricos, más aún cuando el inicio del cuadro es el delirio, que en el fondo tiene una causa orgánica potencialmente letal.

\section{Conflicto de intereses}

Los autores no declaran ningún conflicto de intereses.

\section{Fuente de financiación}

Ninguna.

\section{REFERENCIAS}

1. Young-Fadok TM, Roberts PL, Spencer MP, Wolff BG. Colonic diverticular disease. Curr Probl Surg. 2000;37(7):457-514.

2. Fluxá D, Quera R. Enfermedad diverticular: mitos y realidades. Rev Med Chile. 2017;145(2):209-18. https://doi. org/10.4067/S0034-98872017000200009.

3. Grande G, Zulli C, Bertani H, Mirante VG, Caruso A, Conigliaro R. Endoscopic Treatment of StentRelated Esophagobronchial Fistula. ACG Case Rep J. 2016;3(4):e185. https://doi.org/10.14309/crj.2016.158.

4. Grubbs J, Huerta S. Perforated jejunal diverticulitis in a nonagenarian veteran: A case report. Int J Surg Case Rep. 2017;40:77-9.https://doi.org/10.1016/j.ijscr.2017.09.011.

5. Hevia M, Quera R, Soto L, Regueira T, O’Brien A, Larach A, et al. Diverticulitis aguda de intestino delgado en un paciente con enfermedad de Crohn. Rev Med Chile. 2017;145(3):397401. https://doi.org/10.4067/S0034-98872017000300016.

6. Zager JS, Garbus JE, Shaw JP, Cohen MG, Garber SM. Jejunal diverticulosis: a rare entity with multiple presentations, a series of cases. Dig Surg. 2000;17(6):643-5. https:// doi.org/10.1159/000051978.

7. Dahl C, Crichton M, Jenkins J, Nucera R, Mahoney S, Marx W, et al. Evidence for Dietary Fibre Modification in the Recovery and Prevention of Reoccurrence of Acute, Uncomplicated Diverticulitis: A Systematic Literature Review. Nutrients. 2018;10(2). pii: E137. https://doi. org/10.3390/nu10020137. 
8. López AJ, Ramia JM, De la Plaza R, Alonso S, González JD, Kühnhardt AW. Enfermedad diverticular yeyuno-ileal complicada tratada quirúrgicamente: serie de 12 casos y revisión de literatura. Rev Gastroenterol Peru. 2017;37(3):240-5.

9. Karas L, Asif M, Chun V, Khan FA. Complicated small bowel diverticular disease: a case series. BMJ Case Rep. 2017;2017. pii: bcr-2017-219699. https://doi.org/10.1136/bcr-2017219699.

10. Seymour CW,Liu VX, Iwashyna TJ, Brunkhorst FM, Rea TD, Scherag A, et al. Assessment of Clinical Criteria for Sepsis: For the Third International Consensus Definitions for Sepsis and Septic Shock (Sepsis-3). JAMA. 2016;315(8):762-74. https://doi.org/10.1001/jama.2016.0288.

11. Bejarano M, Gallego CX, Gómez JR. Frecuencia de abdomen agudo quirúrgico en pacientes que consultan al servicio de urgencia. Rev Colomb Cir. 2011;26(1):33-41.

12. Jarral OA, Purkayastha S, Darzi A, Zacharakis E. Education and Imaging. Gastrointestinal: Enterolith-induced perforation on a background of jejunal diverticulum.J Gastroenterol Hepatol. 2010;25(2):429. https://doi.org/10.1111/j.14401746.2010.06261.x.

13. Freeman HJ. Spontaneous free perforation of the small intestine in adults. World J Gastroenterol. 2014;20(29):9990-7. https://doi.org/10.3748/wjg.v20.i29.9990.
14. Ocampo Chaparro JM, González Hadad A. Acute abdomen in the elderly. Rev Colomb Cir. 2006;21(4):266-82.

15. Ocampo JM, Reyes-Ortiz CA, Rengifo A, Velasco MM. Isquemia mesentérica crónica en ancianos: un reto diagnóstico. Rev Colomb Cir. 2017;32:229-35.

16. Kavanagh C, Kaoutzanis C, Spoor K, Friedman PF. Perforated jejunal diverticulum: a rare presentation of acute abdomen. BMJ Case Rep. 2014;2014. pii: bcr-2013-202673. https://doi.org/10.1136/bcr-2013-202673.

17. Johnson KN, Fankhauser GT, Chapital AB, Merritt MV, Johnson DJ. Emergency management of complicated jejunal diverticulosis. Am Surg. 2014;80(6):600-3.

18. Ocampo JM, Osorno DA. Delirium: un gigante de la geriatría. Manizales: Universidad de Caldas; 2009.

19. Peery AF, Barrett PR, Park D, Rogers AJ, Galanko JA, Martin $\mathrm{CF}$, et al. A high-fiber diet does not protect against asymptomatic diverticulosis. Gastroenterology. 2012;142(2):26672.e1. https://doi.org/10.1053/j.gastro.2011.10.035.

20. Durgakeri P, Sarkar A. Perforated jejunal diverticulum: a case report. ANZ J Surg. 2017;87(7-8):634-5. https://doi. org/10.1111/ans.12954. 